\title{
Totality of Evidence Supporting the Use of ABP 980, a Trastuzumab Biosimilar: Practical Considerations
}

\author{
Hans-Christian Kolberg • Georgia Savva Demetriou • Vladimir Hanes
}

Received: May 26, 2020 / Accepted: July 3, 2020 / Published online: January 11, 2021

(C) The Author(s) 2021

\section{ABSTRACT}

ABP 980 (KANJINTI ${ }^{\mathrm{TM}}$, Amgen, Thousand Oaks, CA, USA; Amgen Europe B.V., The Netherlands) is a biosimilar to trastuzumab (Herceptin ${ }^{\circledR}$ ), a monoclonal antibody that selectively binds human epidermal growth factor receptor-2 (HER2). Here we provide a brief overview of the totality of evidence (including analytical [structural and functional] characterization, nonclinical evaluation, and human pharmacokinetic $[\mathrm{PK}]$, pharmacodynamic, and clinical assessment comparing ABP 980 with trastuzumab reference product $[\mathrm{RP}]$ ) that supported the approval of ABP 980, along with practical considerations on the reconstitution and use of the

Electronic supplementary material The online version of this article (https://doi.org/10.1007/s40487020-00129-x) contains supplementary material, which is available to authorized users.

H.-C. Kolberg $(\square)$

Department of Obstetrics and Gynecology, Breast Cancer Center, and Gynecologic Cancer Center, Marienhospital Bottrop GmbH, Josef-Albers-Str. 70, 46236 Bottrop, Germany

e-mail: hans-christian.kolberg@mhb-bottrop.de

G. S. Demetriou

Department of Medical Oncology, University of

Witwatersrand, Johannesburg, South Africa

V. Hanes

Biosimilars Business Unit, Amgen Inc., 1 Amgen

Center Drive, Thousand Oaks, CA 91320, USA lyophilized product to ensure safe and effective administration. ABP 980 has been shown to be highly similar to the RP, with similar mechanism of action, binding, and potency. Key PK parameters, geometric means ratio (GMR [90\% $\mathrm{CI}]$ ) of $C_{\max }$ and $\mathrm{AUC}_{\mathrm{inf}}$, are comparable and within the equivalence margin of 0.80 to 1.25 (ABP 980: 1.04 [0.99-1.08] versus trastuzumab US: 1.06 [1.00-1.12]; ABP 980: 0.99 [0.95-1.03] versus trastuzumab EU: 1.00 [0.95-1.06]). No clinically meaningful differences were found between ABP 980 and RP in a comparative clinical trial in patients with HER2-positive early breast cancer. Pathological complete response-ABP 980: 48\% versus RP: 41\% (risk difference [RD], 90\% CI: 7.3\%, 1.2-13.4; relative risk [RR], 90\% CI: 1.188, 1.033-1.366). Sensitivity analyses per central pathology reviewABP 980: 48\%; RP: 42\% (RD: $5.8 \%,-0.5$ to 12.0 ; RR: 1.142, 0.993-1.312), with RD and RR falling within predefined equivalence margins. Similar to trastuzumab RP, KANJINTI ${ }^{\mathrm{TM}}$ is supplied as a sterile, lyophilized cake to be reconstituted with bacteriostatic water for injection (BWFI) for multiple-dose injection or sterile WFI for single use. Stability data support storage of reconstituted solution at $2-8^{\circ} \mathrm{C}\left(36-46^{\circ} \mathrm{F}\right)$, up to 28 days. Reconstituted solution can be diluted in infusion bags containing $0.9 \%$ saline and stored for up to $24 \mathrm{~h}$ prior to intravenous administration. 
Keywords: ABP 980; Biosimilar; Breast cancer; Gastric cancer; Gastroesophageal adenocarcinoma; Kanjinti; Reconstitution; Switching; Trastuzumab

\section{Key Summary Points}

ABP 980 (KANJINTI $^{\mathrm{TM}}$ ) is a biosimilar to trastuzumab reference product (RP).

It is highly similar to the RP in analytical characteristics and, like the RP, binds with high affinity to human epidermal growth factor receptor-2 (HER2), blocking its activation and inhibiting the proliferation of HER2-expressing cells.

The pharmacokinetics and pharmacodynamics of ABP 980 are also highly similar to those of trastuzumab RP; in a healthy volunteer equivalence study, the geometric mean ratios and 90\% confidence intervals of key PK parameters-AUC $\mathrm{A}_{0 \text {-inf }}$ and $C_{\max }$-for the two products fell within the equivalence criteria of $0.80-1.25$.

A comparative clinical trial in patients with early breast cancer has shown similar pathologic complete response with $\mathrm{ABP}$ 980 versus $R P$, with no new safety or immunologic concerns.

Like the RP, ABP 980 product is supplied as a lyophilized powder that should be reconstituted and diluted prior to use as intravenous infusion.

The totality of evidence showed that ABP 980 is highly similar to trastuzumab RP, with no clinically meaningful differences, thus supporting extrapolation to all approved indications of the RP and providing an alternative to trastuzumab $\mathrm{RP}$ in the treatment of patients with breast and gastric cancers.

\section{DIGITAL FEATURES}

To view digital features for this article go to: https://doi.org/10.6084/m9.figshare.12589316.

\section{INTRODUCTION}

ABP 980 (KANJINTI $^{\mathrm{TM}}$, Amgen, Thousand Oaks, CA, USA; Amgen Europe B.V., The Netherlands) is a biosimilar to trastuzumab (Herceptin ${ }^{\circledR}$, Genentech, South San Francisco, CA, USA; Roche Registration GmbH, Grenzach-Wyhlen, Germany), an anti-human epidermal growth factor receptor-2 (HER2) monoclonal antibody $[1,2]$. ABP 980 is approved in the United States, the European Union, and Japan for all approved indications of the trastuzumab reference product (RP), including the treatment of HER2-overexpressing breast cancer, metastatic gastric cancer, and gastroesophageal junction adenocarcinoma [1-3].

Biosimilars are biologics that are highly similar to their reference or originator biologics. While the exact definitions vary (Table 1 ), regulatory authorities worldwide use the same underlying principles to define biosimilars as biologic agents that are highly similar to their originator product without any clinically meaningful differences in function, potency, purity, pharmacokinetics (PK), pharmacodynamics (PD), and clinical efficacy, safety, and immunogenicity, with allowance for minor differences in clinically inactive components [4]. Biosimilars are expected to be developed and approved according to rigorous standards of pharmaceutical quality and safety based on a distinct regulatory pathway that is different from that for originator biologics and smallmolecule generic drugs, and are expected to provide an alternative to existing biologic agents and expand therapeutic options and treatment choices $[4,5]$.

Biosimilars are not generics. Generic drugs are small, chemically synthesized molecules 
Table 1 Worldwide regulatory definitions of biosimilars [4]

\begin{tabular}{|c|c|c|}
\hline Regulatory authority & Definition & Reference \\
\hline $\begin{array}{l}\text { The European Medicines } \\
\text { Agency (EMA) }\end{array}$ & $\begin{array}{l}\text { A biosimilar is a biological medicine highly } \\
\text { similar to another already approved } \\
\text { biological medicine (the 'reference medicine') } \\
\text { (Biosimilars are approved according to the } \\
\text { same standards of pharmaceutical quality, } \\
\text { safety, and efficacy that apply to all biological } \\
\text { medicines) }\end{array}$ & $\begin{array}{l}\text { The European Medicines Agency. } \\
\text { Biosimilar Medicines }\end{array}$ \\
\hline $\begin{array}{l}\text { US Food and Drug } \\
\text { Administration (US- } \\
\text { FDA) }\end{array}$ & $\begin{array}{l}\text { A biosimilar is a biologic product that is highly } \\
\text { similar to a US-licensed reference biological } \\
\text { product, notwithstanding minor differences } \\
\text { in clinically inactive components, and for } \\
\text { which there are no clinically meaningful } \\
\text { differences between the biological product } \\
\text { and the RP in terms of the safety, purity, and } \\
\text { potency of the product }\end{array}$ & $\begin{array}{l}42 \text { U.S. Code } \$ 262(\mathrm{i})(2)-\text { Regulation of } \\
\text { biological products } \\
\text { FDA Guidance for Industry: Questions } \\
\text { and Answers regarding BPCIA (2015) }\end{array}$ \\
\hline $\begin{array}{l}\text { The Japanese } \\
\text { Pharmaceuticals and } \\
\text { Medical Devices Agency } \\
\text { (Jp-PMDA) }\end{array}$ & $\begin{array}{l}\text { A biosimilar is a follow-on biological medicinal } \\
\text { product (FOBMP) that is a new } \\
\text { biotechnological medicinal product } \\
\text { developed to be similar to an already } \\
\text { licensed, biotechnology medical product } \\
\text { (reference biological product; RBP) and is } \\
\text { developed on the basis of data that } \\
\text { demonstrate comparability between the } \\
\text { FOBMP and the RBP with respect to } \\
\text { quality, safety, and efficacy or other relevant } \\
\text { data }\end{array}$ & $\begin{array}{l}\text { Japan Generic Medicines Association Nov } \\
\text { 25, } 2010 \text { Interim Translation of } \\
\text { Notification LED, PFSB, MHLW; } \\
\text { Yakushokushinsa No. } 0304007 \text { (Mar 4, } \\
\text { 2009) }\end{array}$ \\
\hline $\begin{array}{l}\text { The World Health } \\
\text { Organization (WHO) }\end{array}$ & $\begin{array}{l}\text { A biosimilar is a similar biotherapeutic product } \\
(\mathrm{SBP}) \text {, defined as "a biotherapeutic product, } \\
\text { which is similar in terms of quality, safety, } \\
\text { and efficacy to an already licensed reference } \\
\text { biotherapeutic product" }\end{array}$ & $\begin{array}{l}\text { WHO Guidelines on Evaluation of Similar } \\
\text { Biotherapeutic Products (2009) }\end{array}$ \\
\hline
\end{tabular}

identical to their RPs, while biosimilars are large biologic agents that are more complex, synthesized in living systems using complex processes, and although similar to their RPs, not identical to them. Their production is inherently dependent on factors such as the characteristics and growing conditions of the cells producing them, complex purification processes (e.g., viral filtration), and storage conditions, which independently and variably influence their structure and stability [6]. Thus, biosimilars should be considered as unique but highly similar molecules to their originator RPs. To ensure that these differences do not negatively impact efficacy, safety, and immunogenicity, a proposed biosimilar must undergo extensive and robust 


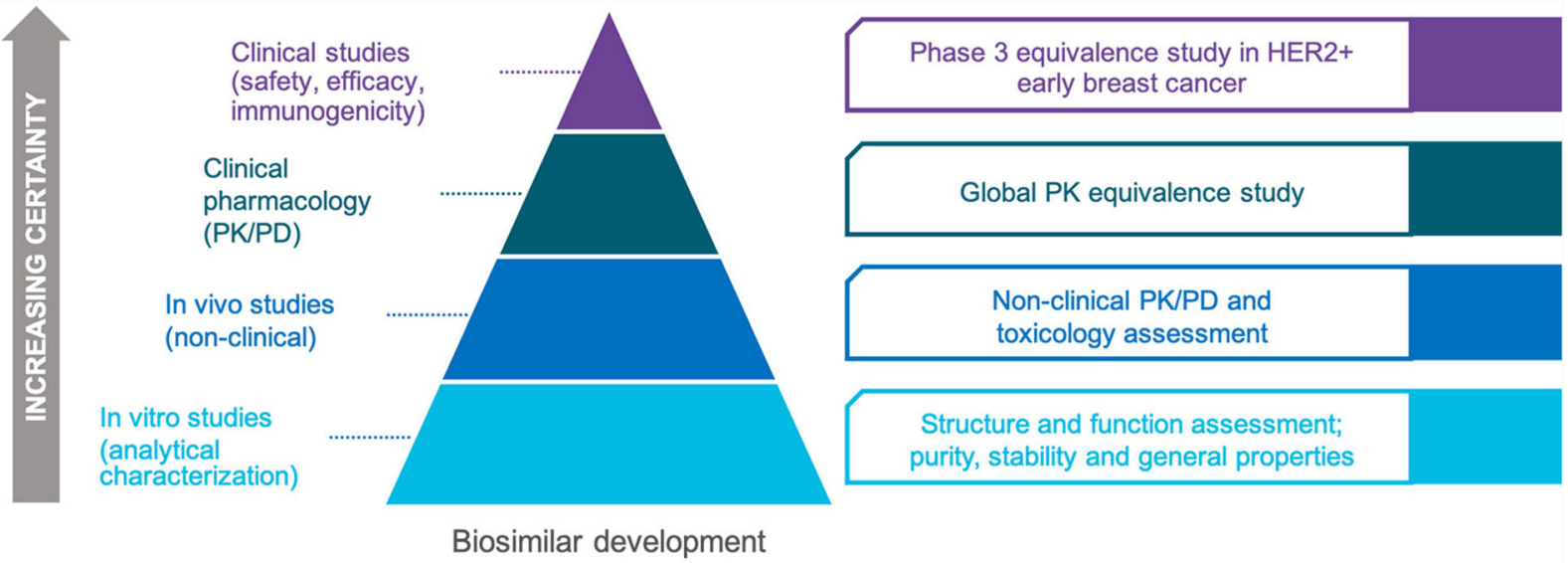

HER2+, human epidermal growth factor receptor 2 positive; $P D$, pharmacodynamics; $P K$, pharmacokinetics

Fig. 1 Illustration depicting a stepwise totality of evidence (TOE) approach for demonstration of biosimilarity of ABP 980 to trastuzumab reference product [13]

scientific investigation. This involves a stepwise process that starts with comprehensive analytical (i.e., structural and functional) characterization, followed by preclinical evaluation including mechanism of action (MOA) and human PK and PD studies, and then a comparative clinical study to evaluate efficacy, safety, and immunogenicity in a representative indication in a sensitive population using sensitive endpoints [7]. It is this "totality of evidence" (TOE) that forms the basis of approval of a proposed biosimilar (Fig. 1).

Biosimilars are typically approved for all requested indications that the RP is approved for based on the TOE generated during the development process. Unlike originator products, regulatory pathways for biosimilars do not require clinical studies in every indication. Approval for indications that are not evaluated clinically is sought using the concept of extrapolation that is unique to biosimilars. Extrapolation should be supported by scientific justification (e.g., similarity in MOA, structural and functional attributes, similar PK/PD, safety profiles, immunogenicity) [5, 7]. This article is based on previously conducted studies and does not contain any new studies with human participants or animals performed by any of the authors.

\section{TOTALITY OF EVIDENCE FOR ABP 980: A BRIEF REVIEW}

As discussed above, analytical (structural and functional) characterization is the first and foundational step in the determination of biosimilarity and begins with the identification of critical quality attributes that may influence the PK, efficacy, safety, and immunogenicity of the proposed biosimilar $[5,8]$. A comprehensive analytical characterization of ABP 980 was conducted using state-of-the-art techniques to identify any potential differences that may exist in structure and function between ABP 980 and trastuzumab RP [9], and demonstrated high analytical similarity of the two in physicochemical characteristics (primary structure/ amino acid sequence, post-translational modifications, higher-order structure, protein content, and product-related impurities and substances) $[9,10]$. ABP 980 has the same amino acid sequence and molecular mass (consistent with theoretical mass), and the same N-linked glycosylation at $\mathrm{N}^{300}$ in the same glycopeptide, as trastuzumab RP. No new peaks or missing peaks were noted in the chromatograms or peptide maps of ABP 980 versus trastuzumab RP. A comparison of the glycan profiles of ABP 980 and trastuzumab RP indicated that while the 
levels of specific species of glycans (e.g., A1G0F) in each may differ, the overall levels of key glycan groups (e.g., afucosylated, galactosylated, sialylated, and high mannose species) that have the ability to influence binding to the Fc $\gamma$ RIIIa receptor and affect antibody-dependent cellular toxicity (ADCC) were similar in both. Moreover, the predicted NK92 ADCC activity for any given glycan combination was found to be similar for ABP 980 and trastuzumab RP. There were no new peaks in the ABP 980 glycan map compared with that of trastuzumab RP.

Likewise, functional similarity evaluations of ABP 980 and trastuzumab RP assessed Fab-mediated, Fc-mediated, and combined Fab- and Fcmediated activities. Since the biological activity of trastuzumab is primarily mediated via binding to the extracellular domain of HER2 and subsequent internalization and activation of downstream ligand-independent signaling events, evaluation of these parameters showed that ABP 980 exhibited similar binding to the extracellular domain of HER2, similar potency, and similar antibody-dependent cellular cytotoxicity activities in experimental cell-based systems [9-11].

ABP 980 Mechanism of Action Animation (MPG $2115141 \mathrm{~kb})$

ABP 980 and trastuzumab RP exhibited similar on-rate, off-rate, and relative affinity for the extracellular domain of the HER2 receptor as measured with surface plasmon resonance, with all values for ABP 980 being contained within the ranges of the RP. A competitive cell binding assay using SK-BR-3 breast cancer cells was conducted comparing ABP 980 and trastuzumab $\mathrm{RP}$ which showed that the mean relative HER2 cell binding of all lots and products tested was approximately $100 \%$, confirming similar binding activity of the two. Post-binding HER2 internalization documented by flow cytometric analysis of HER2-expressing SK-BR-3 cells treated with ABP 980 and trastuzumab RP confirmed similar levels of internalization of the two antibodies. Finally, similar inhibition of proliferation with ABP 980 and RP in NCI-N87 gastric cancer cells expressing high HER2 levels and similar lack of inhibition of proliferation in MCF7 breast cancer cells expressing low levels of HER2 confirmed biosimilarity of ABP 980 with trastuzumab RP as well as ABP 980 activity in gastric cancer cells and specificity of ABP 980 for HER2-overexpressing cells. Further, similar synergistic activity in the presence of the chemotherapeutic agent docetaxel was demonstrated in combination studies using gastric cancer cells in vitro [12]. Nonclinical pharmacology evaluations have established that $\mathrm{ABP}$ 980 and trastuzumab RP have similar dose-dependent antitumor activity in tumor xenograft models (breast or gastric cancer cells) that overexpressed HER2, and multiple-dose toxicology studies in cynomolgus monkeys showed similar in vivo drug exposure and dose accumulation $[11,13]$.

The clinical pharmacology of ABP 980 was evaluated in a randomized, single-blind, singledose PK equivalence study in healthy subjects. This study confirmed the PK similarity between ABP 980 and trastuzumab RP (Table 2) [14]. Additionally, the percentage of treatment-related adverse events (AEs) of all grades was similar in both groups, and immunogenicity evaluations revealed that no patients developed antidrug antibodies during the study.

Finally, clinical safety and efficacy of ABP 980 were demonstrated in a randomized, multicenter, double-blind, comparative clinical study (LILAC) conducted in patients with early breast cancer (EBC) $[15,16]$. This patient population is considered to be a sensitive population in which to evaluate a trastuzumab biosimilar because of its homogeneity and minimal confounding factors such as prior treatments that may confound immunogenicity evaluation, line of therapy, disease burden, comorbidities, and risk of secondary cancers [17-19]. These characteristics make it more suitable for an equivalence study between two similar products such as a biosimilar and its RP.

A total of 725 patients were randomized in the LILAC study to receive ABP $980(n=364)$ or trastuzumab RP $(n=361)$ plus paclitaxel $\left(175 \mathrm{mg} / \mathrm{m}^{2}\right.$ every 3 weeks or $80 \mathrm{mg} / \mathrm{m}^{2}$ once weekly for 12 cycles if that was the standard of care in that region) followed by surgery. The coprimary efficacy endpoints in this study were risk difference (RD) and risk ratio (RR) of pathological complete response (pCR) in breast 
Table 2 Statistical assessment of pharmacokinetic parameters [14]

\begin{tabular}{|c|c|c|c|}
\hline & \multicolumn{3}{|l|}{ PK parameters } \\
\hline & $C_{\max }(\mu \mathrm{g} / \mathrm{mL})$ & $\mathrm{AUC}_{\text {inf }}(\mu \mathrm{g} \cdot \mathrm{h} / \mathrm{mL})$ & $\operatorname{AUC}_{\text {last }}(\mu \mathrm{g} \cdot \mathrm{h} / \mathrm{mL})$ \\
\hline \multicolumn{4}{|l|}{ Adjusted LS GM $(n)$} \\
\hline ABP 980 & $135.9(50)$ & $34,061.4(50)$ & $33,811.7(50)$ \\
\hline Trastuzumab (US) & $131.2(52)$ & $32,271.7(48)$ & $32,113.6(48)$ \\
\hline Trastuzumab (EU) & $136.8(54)$ & $33,947.0(46)$ & $33,748.2(46)$ \\
\hline \multicolumn{4}{|c|}{ Statistical analysis: ratio and $90 \% \mathrm{CI}$ of adjusted least square geometric means } \\
\hline ABP 980 versus trastuzumab (US) & $1.04(0.9948-1.0787)$ & $1.06(0.9974-1.1169)$ & $1.05(0.9967-1.1122)$ \\
\hline ABP 980 versus trastuzumab (EU) & $0.99(0.9540-1.0338)$ & $1.00(0.9476-1.0624)$ & $1.00(0.9479-1.0589)$ \\
\hline $\begin{array}{l}\text { Trastuzumab (US) versus trastuzumab } \\
(\mathrm{EU})\end{array}$ & $0.96(0.9213-0.9975)$ & $0.95(0.8973-1.0072)$ & $0.95(0.8998-1.0063)$ \\
\hline
\end{tabular}

Statistical model includes treatment and ethnicity as fixed effects

$G M$ geometric means, $L S$ least squares, $n$ number of non-missing observations

tissue and axillary lymph nodes assessed at a local laboratory $[15,16]$. A central laboratory performed a sensitivity analysis of tumor samples in order to reduce inter-pathologist variability in response determination. Based on local evaluation, pCR was achieved in 172/358 assessable patients $(48 \%, 90 \%$ CI $43-53 \%)$ in the ABP 980 group and $137 / 338$ patients $(41 \%$, 90\% CI 35-46\%) in the trastuzumab RP group (RD 7.3\%, 90\% CI 1.2-13.4; RR 1.188, 90\% CI 1.033-1.366); the upper bounds of CIs exceeded predefined equivalence margins of $13 \%$ for RD and of 1.318 for RR (Fig. 2). The pCR was noted in 162/339 (48\%) patients in the ABP 980 group at baseline and $138 / 330$ (42\%) patients in the trastuzumab RP group at baseline (RD 5.8\%, $90 \% \mathrm{CI}-0.5$ to 12.0 , and RR $1.142,90 \% \mathrm{CI}$ 0.993-1.312) in central lab assessment. Based on these sensitivity analyses, similar efficacy of ABP 980 and trastuzumab RP was confirmed, as the RR and RD estimates for the two groups were contained within the predefined equivalence margins of the study.

In the LILAC study, after neoadjuvant treatment and surgery, patients received adjuvant treatment with ABP 980 or RP every 3 weeks for up to 1 year from start of study treatment. During the adjuvant phase of the study, patients who had previously received ABP 980 continued with ABP 980 (6 mg/kg); patients who had previously received trastuzumab RP either continued to receive trastuzumab RP or were switched to receive ABP 980. The overall type, frequency, and severity of AEs and AEs of interest were similar between the ABP 980 and trastuzumab RP during both the neoadjuvant and adjuvant phases of the clinical study (Fig. 3) [15]. There was no difference in the incidence of AEs among patients who were switched from the RP to ABP 980 and those who continued to receive trastuzumab RP in the adjuvant setting. Median left ventricular ejection fraction (LVEF), a measure of cardiotoxicity, did not change in any treatment group over the course of the study, and switching had no impact on LVEF (Fig. 4). The overall frequency of cardiac disorders was low throughout the study $[13,15,20]$. The frequency of immunogenicity was found to be low and similar between groups, and no patient tested positive for neutralizing antibodies [15].

Based on the sum of these results, the TOE for ABP 980 confirms that it is highly similar to trastuzumab RP, thereby supporting extrapolation to all approved indications of the RP [13]. 

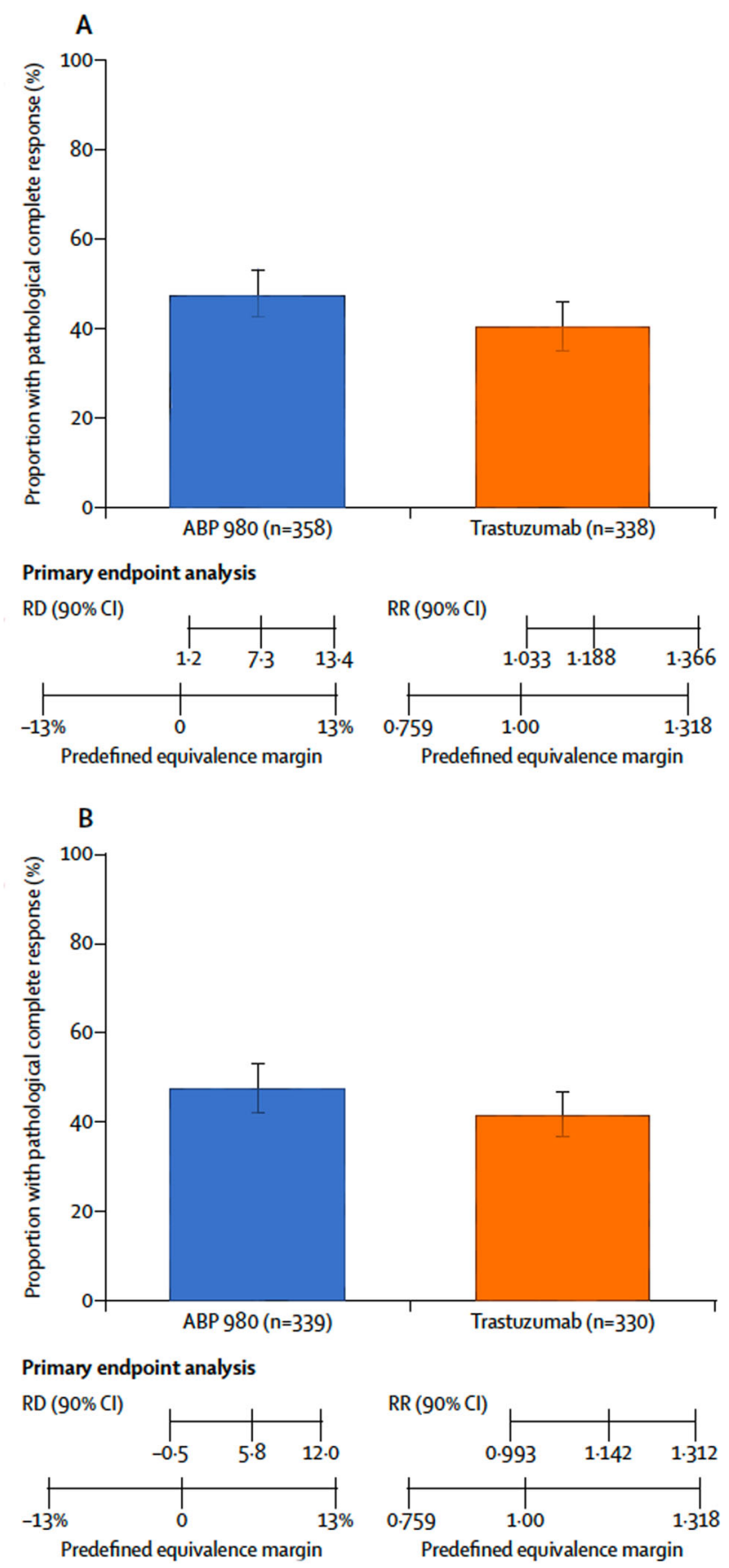

Fig. 2 Proportion of patients with pathological complete response (pCR) based on (a) local laboratory review and (b) central laboratory review. PCR was defined as the absence of invasive tumor cells in the breast tissue and axillary lymph node(s) regardless of residual ductal carcinoma in situ (DCIS) [15]. $R D$ risk difference, $R R$ risk ratio 
Treatment-Emergent Adverse Events of Interest in the Entire Study (Safety Analysis Population; Grade $\geq 3$ )

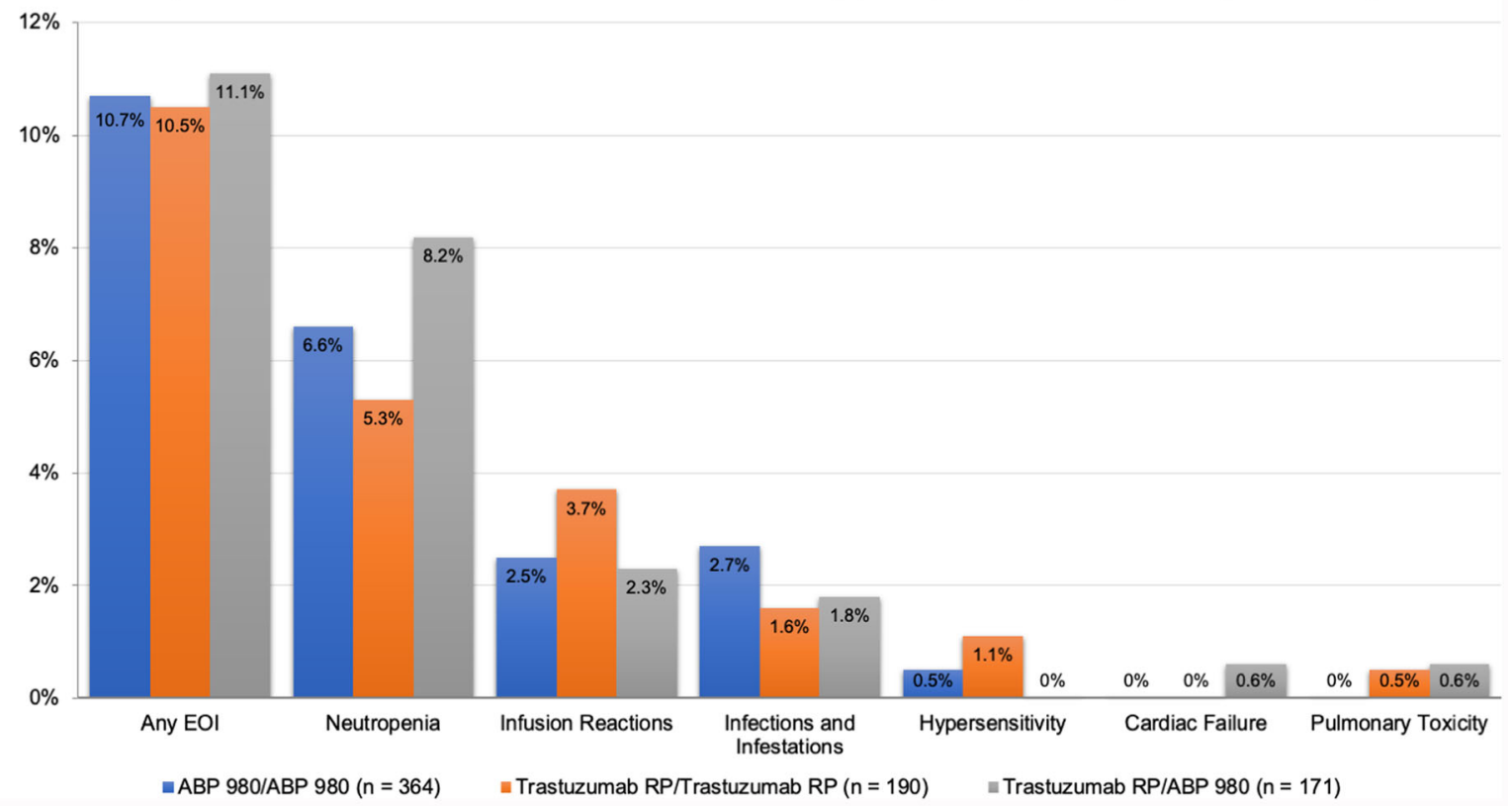

Fig. 3 Treatment-emergent adverse events of interest in the entire study (safety analysis population; grade $\geq 3$ ). EOI event of interest, $R P$ reference product

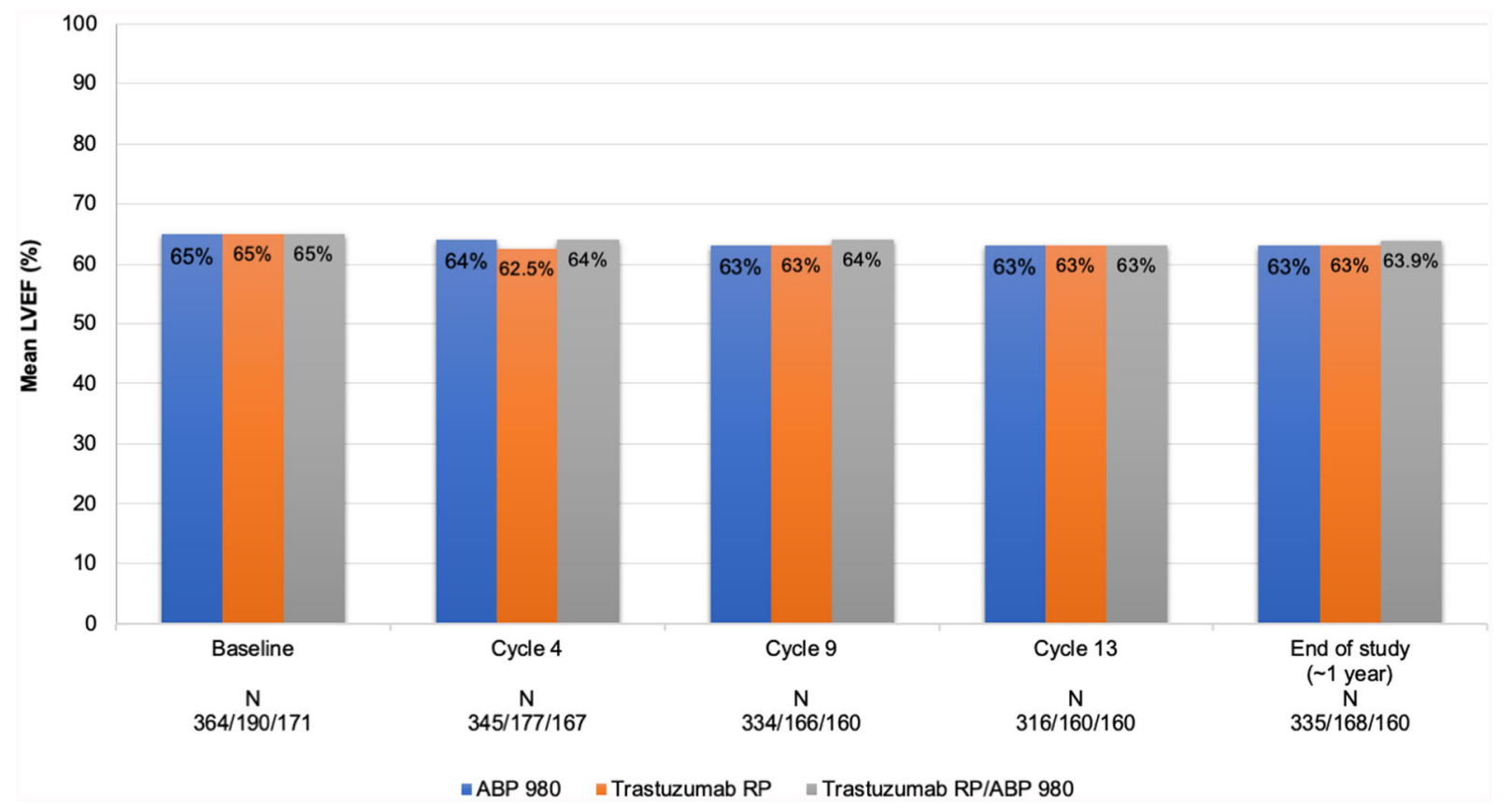

Fig. 4 Left ventricular ejection fraction in the entire study [20]. LVEF left ventricular ejection fraction 
Table 3 Preparation and use of ABP $980[1,2]$

1. Preparing for administration 1.1 First, check the vial label to confirm that the drug being prepared and administered is ABP 980 and not another intravenous (IV) formulation (e.g., ado-trastuzumab emtansine, an antibody drug conjugate). This is an important step to prevent medication errors.

Each glass vial contains either $150 \mathrm{mg}$ or $420 \mathrm{mg}$ of active ingredient.

1.2 Next, visually examine the vial and contents of the vial. The ABP 980 product for injection is a sterile lyophilized powder with a white to pale yellow color and cake-like appearance.

1.3 It should be reconstituted and further diluted before administering as an IV infusion.

$1.4 \mathrm{It}$ is also preservative-free and therefore should be appropriately prepared and stored, as necessary, under specific conditions before IV administration $[1,2]$

2. Reconstitution general considerations
3. Dilution
2.1 Reconstitute each 420-mg vial of the ABP 980 product with $20 \mathrm{~mL}$ of bacteriostatic water for injection (BWFI) USP, containing $0.9 \%$ to $1.1 \%$ benzyl alcohol as a preservative. This will result in a multiple-dose solution of $20 \mathrm{~mL}$ containing $21 \mathrm{mg} / \mathrm{mL}$ ABP 980

If you are using the $150-\mathrm{mg}$ vial, reconstitute with $7.2 \mathrm{~mL}$ of sterile water for injection (SWFI) to yield a $21-\mathrm{mg} / \mathrm{mL}$ solution for single use

In patients with known hypersensitivity to benzyl alcohol, reconstitute the ABP 980 product with $20 \mathrm{~mL}$ of SWFI. Note that SWFI does not contain any preservative. Therefore, reconstitution with SWFI only yields a single-use solution

2.2 After reconstitution with BWFI, the solution may be stored in the vial for up to 28 days at $2-8{ }^{\circ} \mathrm{C}$ $\left(36-46^{\circ} \mathrm{F}\right)$; however, after reconstitution with SWFI, the solution must be used immediately

2.3 After reconstitution, the ABP 980 can be further diluted in an IV bag and administered as an IV infusion

2.4 Please note that it is always necessary to use appropriate aseptic technique when performing the reconstitution steps:

Take a sterile syringe and slowly inject the $20 \mathrm{~mL}$ of BWFI (or SWFI in the case of patients with known hypersensitivity to benzyl alcohol), into the vial containing the lyophilized powder of ABP 980. The stream of BWFI from the syringe should be aimed at the lyophilized cake at the bottom of the vial

Then, gently swirl the vial so that the powder mixes with the diluent. This facilitates reconstitution. A note of caution-DO NOT SHAKE the vial vigorously at this time

Once the solution is mixed or reconstituted, a slight foaming may be visible. If so, allow the vial to stand undisturbed and settle down for about $5 \mathrm{~min}$

Handle the ABP 980 carefully during reconstitution. Again, do not shake the reconstituted solution vigorously as this will result in excessive foaming and may prevent withdrawal of the correct amount of ABP 980 from the vial

As with any parenteral drug product, inspect the vial visually for particulate matter and for any signs of discoloration before moving to dilution and IV infusion

The ABP 980 reconstituted solution should appear free of any particulate matter, be clear to slightly opalescent, and colorless to pale yellow

Store this reconstituted solution in a refrigerator at $2-8{ }^{\circ} \mathrm{C}\left(36-46^{\circ} \mathrm{F}\right)$. Do not freeze after reconstitution and discard any solution that is unused after 28 days

Note that if the ABP 980 powder is reconstituted with SWFI without preservative, it should be used immediately, and any unused portion of this solution should be discarded right way

3.1 The reconstituted solution can be used immediately for IV infusion (or stored as indicated above and used within 28 days if reconstituted with BWFI)

3.2 To do this, calculate the volume of the $21-\mathrm{mg} / \mathrm{mL}$ reconstituted ABP 980 solution needed, withdraw this volume from the vial, and add it to an infusion or IV bag containing $250 \mathrm{~mL}$ of $0.9 \%$ Sodium Chloride Injection, USP. The volume to be withdrawn will be based on the dose required for each patient (as defined in the package insert)

Again, a note of caution-Do not use an IV bag with dextrose (5\%) solution at this step. Only use an IV bag containing $0.9 \%$ Sodium Chloride Injection, USP

3.3 Once the ABP 980 solution is added to the IV bag, gently mix the contents by inverting the bag back and forth

This solution of ABP 980 that is diluted in polyvinylchloride (PVC) or polyethylene (PE) IV bags containing $0.9 \%$ Sodium Chloride Injection, USP, is now ready for infusion. It can be stored at $2-8{ }^{\circ} \mathrm{C}\left(36-46^{\circ} \mathrm{F}\right)$ for up to but not more than $24 \mathrm{~h}$ prior to use. This storage time is specific to the IV bag with ABP 980 solution and is additional to the time allowed for reconstitution and storage of reconstituted vial. Note: Do not freeze this solution 


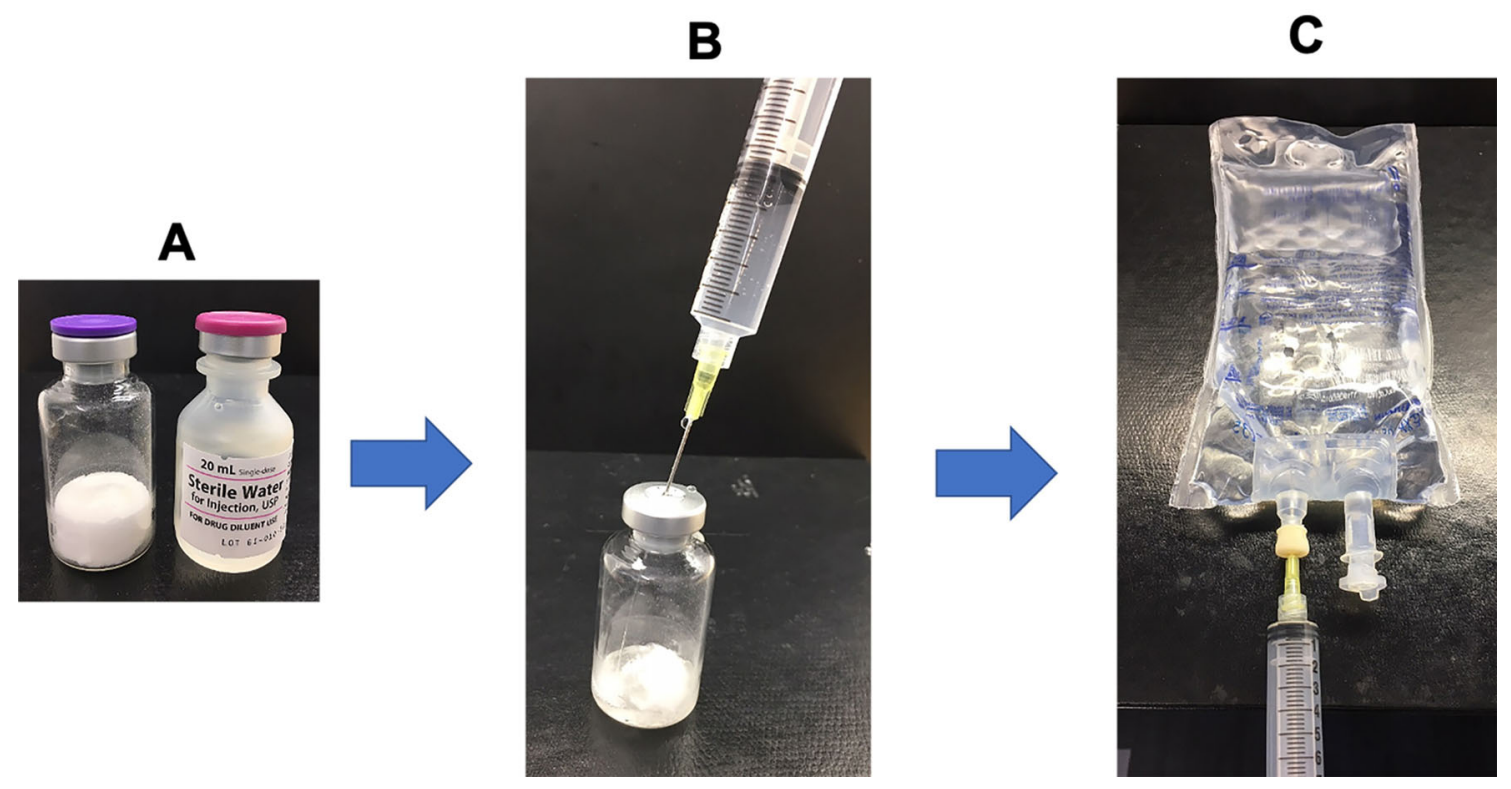

Fig. 5 Reconstitution and dilution of ABP 980 product. a Lyophilized ABP 980 drug product and SWFI diluent. b Reconstitution of lyophilized drug product. c Dilution in $250 \mathrm{~mL} 0.9 \%$ saline IV bags

\section{USE OF ABP 980: PRACTICAL CONSIDERATIONS}

The results from the stepwise development studies reviewed in the preceding section provide robust scientific evidence to support the similarity of ABP 980 to trastuzumab RP with no

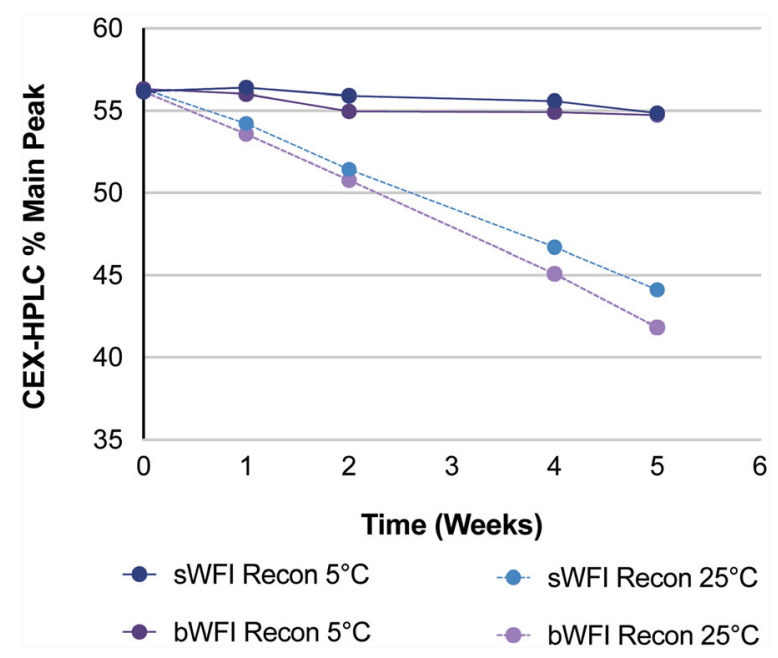

Fig. 6 Reconstituted ABP 980 product: percent of main peak (intact molecule) by cation exchange high performance liquid chromatography (CEX-HPLC) clinically meaningful differences, and support its use as an alternative treatment option to trastuzumab RP for patients with breast and gastric cancers [4]. Indeed, ABP 980 (KANJIN$\mathrm{TI}^{\mathrm{TM}}$ ) is now approved in the United States, European Union, and Japan for all approved indications of the RP (Herceptin ${ }^{\circledR}$ ), i.e., treatment of HER2+ metastatic breast cancer, EBC, and metastatic gastric cancer. The use of biosimilars such as ABP 980 expand treatment options for patients.

Let us now examine the practical considerations associated with administration of the ABP 980 drug product to patients. Like trastuzumab RP, ABP 980 is supplied as a lyophilized powder and should be administered intravenously after reconstitution and further dilution $[1,2]$. Table 3 provides a step-by-step protocol for the preparation, reconstitution, and dilution of the ABP 980 product.

Preparation and Use of ABP 980 Video (MPG $288970 \mathrm{~kb}$ )

Figure 5 shows the various materials used for reconstitution and dilution. While general precautions related to the preparation and administration of intravenous (IV) products (e.g., confirming product strength, inspecting visual appearance, checking dosage calculations, using 


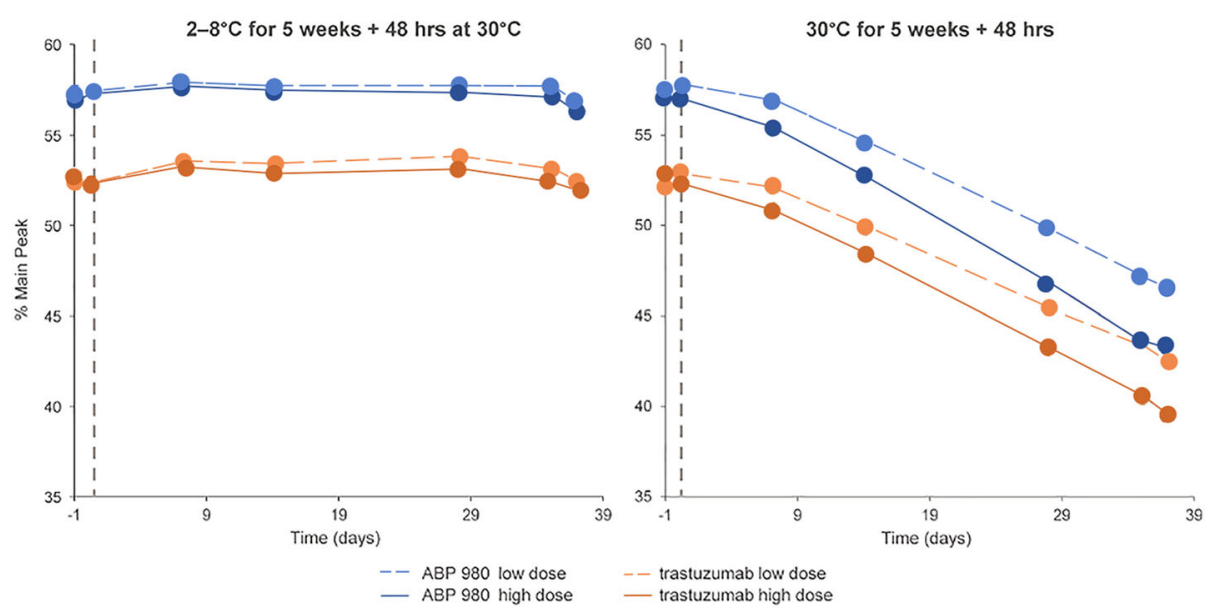

Fig. 7 Purity analysis of main peak by CEX-HPLC upon exposure to $30{ }^{\circ} \mathrm{C}$ (Crampton et al. [23])

aseptic technique) should be followed at all times, there are a few precautions specific to the use of ABP 980 as with the use of the RP. Thus, bacteriostatic water for injection (BWFI) should be generally used for reconstitution of ABP 980 . When sterile water for injection (SWFI) is used, as indicated for patients with known hypersensitivity to BWFI, the reconstituted solution should be used immediately and not stored. Second, care should be taken not to vigorously shake the vial during reconstitution of the lyophilized powder with diluent, as this can cause foaming and interfere with withdrawal of the correct dose from the vial when preparing for dilution in an IV bag. It is best to let the reconstituted solution stand for $5 \mathrm{~min}$ after gentle mixing. Third, dilution of the reconstituted solution may be done in polyvinylchloride or polyethylene infusion bags containing 0.9\% Sodium Chloride Injection, USP; IV bags containing 5\% dextrose solution should not be used for this purpose. Finally, neither reconstituted solutions nor prepared IV bags should be frozen.

When the product is correctly reconstituted with BWFI as described above (and in Table 3), the solution may be stored in the vial for up to 28 days at $2-8{ }^{\circ} \mathrm{C}$; however, after reconstitution with SWFI, the solution must be used immediately, since SWFI does not contain preservative. The guidance for these storage conditions is supported based on stability findings for the reconstituted solution [21]. During the assessment of post-reconstitution stability based on the known potential degradation pathways for trastuzumab RP, it was shown that during storage at $5{ }^{\circ} \mathrm{C}$, SWFI- and BWFI-reconstituted ABP 980 were chemically stable (Fig. 6). However, when the reconstituted ABP 980 solution was exposed to $25^{\circ} \mathrm{C}$ or room temperature, the solution, similar to trastuzumab RP solution, was sensitive to chemical degradation [22]; hence the recommendation to store the reconstitution under refrigeration. Other studies have separately shown that after reconstitution with either diluent, SWFI or BWFI, there was no significant change in the percentage of high molecular weight species or in the percent purity for up to 5 weeks at $5{ }^{\circ} \mathrm{C}$ and $25^{\circ} \mathrm{C}$; there was also no change in subvisible particle counts or visual appearance under these conditions [21]. Thus, reconstitution with either diluent is acceptable. However, as noted previously, SWFIreconstituted solution should be used immediately, as it does not contain preservative.

A drug stability study simulating conditions in a real-world hospital pharmacy setting has evaluated the quality and stability of ABP 980 versus trastuzumab RP solutions after dilution in IV bags [23]. This study investigated the effect of mechanical stress and extended storage for 35 days at $2-8{ }^{\circ} \mathrm{C}$ versus $30^{\circ} \mathrm{C}$, followed by $30^{\circ} \mathrm{C}$ for $48 \mathrm{~h}$ (similar to what may happen when IV bags are prepared ahead of time and stored until use) on product quality, purity, and activity before and after infusion. Based on the 
results of this study, there was no clinically meaningful impact on product stability or quality over extended storage periods of 35 days at $2-8{ }^{\circ} \mathrm{C}$ followed by exposure to $30^{\circ} \mathrm{C}$ for $48 \mathrm{~h}$. Results were consistent for high(1200 mg; $3.8 \mathrm{mg} / \mathrm{mL}$ ) or low-dose (70 mg; $0.3 \mathrm{mg} / \mathrm{mL}$ ) solutions covering the range of potential real-world use. Cation exchange highperformance liquid chromatography analysis of reconstituted ABP 980 and RP demonstrated no significant loss in the main peak (intact molecule) (Fig. 7). However, after exposure to $30^{\circ} \mathrm{C}$ for 5 weeks and $48 \mathrm{~h}, \mathrm{ABP} 980$, like trastuzumab $\mathrm{RP}$, was sensitive to chemical degradation as expected during storage at room temperature [22].

\section{CONCLUSIONS}

Based on the overall TOE including analytical (structural and functional), nonclinical PK/PD, clinical PK, and comparative clinical safety and efficacy studies, ABP 980 has been shown to be highly similar to trastuzumab RP. In the comparative clinical (LILAC) study, no clinically meaningful differences in efficacy, safety, and immunogenicity between ABP 980 and trastuzumab RP were seen. These results supported justification for extrapolation, and subsequent approval for use in all indications for which the trastuzumab RP is approved.

Central to the appropriate use of biosimilars is the understanding of the TOE accrued during development and an understanding of when and how to use specific biosimilars. Here, we have provided an overview of data supporting the approval of ABP 980, a trastuzumab biosimilar, and reviewed the protocol for the proper reconstitution, dilution, storage, and administration of this agent. It is important to note that when reconstituted correctly with BWFI, the ABP 980 product remains stable for 5 weeks at $5{ }^{\circ} \mathrm{C}$ with no increase in visible particles, no chemical degradation, no change in the percentage of high molecular weight species, and no significant change in purity, thereby providing a flexible dosing schedule and prolonged shelf life after reconstitution $[21,23]$. It is expected that these data will contribute to improving the comfort level for its use among healthcare providers.

\section{ACKNOWLEDGEMENTS}

Funding. The studies related to ABP 980 and the Rapid Service Fee were supported by Amgen Inc., Thousand Oaks, California, USA.

Medical Writing, Editorial, and Other Assistance. Medical writing assistance was provided by Saudha Parthasarathy, PhD, from Innovation Communications Group Inc, New York, NY, under the guidance of Monica Ramchandani, PhD, Amgen Inc. Editorial assistance and digital asset support was provided by Shirley Markant, PhD, Patricia Rymer, and Jessica A. Martin from Innovation Communications Group Inc., New York, NY, USA.

Authorship. All named authors meet the International Committee of Medical Journal Editors (ICMJE) criteria for authorship for this article, take responsibility for the integrity of the work as a whole, and have given their approval for this version to be published.

Disclosures. Hans-Christian Kolberg declares that he received honoraria from Carl Zeiss Meditec, TEVA, Theraclion, Novartis, Amgen, AstraZeneca, Pfizer, Janssen-Cilag, GSK, LIV Pharma, Roche and Genomic Health, and non-financial support from Carl Zeiss Meditec, Novartis, Pfizer, Amgen, Roche, LIV Pharma, Tesaro, Daiichi Sankyo, and Genomic Health. Georgia Savva Demetriou is a consultant for AstraZeneca and Celgene and was on speakers' bureaus for Merck and Serono. Vladimir Hanes is an employee and stockholder of Amgen, Inc.

Compliance with Ethics Guidelines. This article is based on previously conducted studies and does not contain any studies with human participants or animals performed by any of the authors.

Open Access. This article is licensed under a Creative Commons Attribution- 
NonCommercial 4.0 International License, which permits any non-commercial use, sharing, adaptation, distribution and reproduction in any medium or format, as long as you give appropriate credit to the original author(s) and the source, provide a link to the Creative Commons licence, and indicate if changes were made. The images or other third party material in this article are included in the article's Creative Commons licence, unless indicated otherwise in a credit line to the material. If material is not included in the article's Creative Commons licence and your intended use is not permitted by statutory regulation or exceeds the permitted use, you will need to obtain permission directly from the copyright holder. To view a copy of this licence, visit http:// creativecommons.org/licenses/by-nc/4.0/.

\section{REFERENCES}

1. KANJINTI ${ }^{\mathrm{TM}}$ (trastuzumab-anns) for injection, for intravenous use. Prescribing information. Amgen Inc., Thousand Oaks, CA: June 2019. https://www. pi.amgen.com/ /media/amgen/repositorysites/piamgen-com/kanjinti/kanjinti_pi.ashx. Accessed 4 Nov 2019.

2. KANJINTI. Summary of product characteristics. Amgen Europe B.V., the Netherlands: September 2018. https://www.ema.europa.eu/en/medicines/ human/EPAR/kanjinti\#productinformation-section. Accessed 4 Nov 2019.

3. Generics and Biosimilars Initiative $(\mathrm{GaBi})$ Online. Biosimilars approved in Japan: 14 December, 2018. http://www.gabionline.net/Biosimilars/General/Bio similars-approved-in-Japan. Accessed 5 May 2020.

4. Thill M, Thatcher N, Hanes V, Lyman GH. Biosimilars: what the oncologist should know. Future Oncol. 2019;15(10):1147-65.

5. Curigliano G, O'Connor DP, Rosenberg JA, Jacobs I. Biosimilars: extrapolation for oncology. Crit Rev Oncol Hematol. 2016;104:131-7.

6. Nathan JJ, Ramchandani M. Kaur P. In: Yamauchi PS, editor. Biologic and systemic agents in dermatology. New York, NY: Springer Publishing Company; 2018. p. 101-10.

7. U.S. Department of Health and Human Services. Scientific considerations in demonstrating biosimilarity to a reference product. Guidance for industry. 2015. https://www.fda.gov/regulatoryinformation/search-fda-guidance-documents/scien tific-considerations-demonstrating-biosimilarityreference-product. Accessed 12 Mar 2020.

8. Markus R, Liu J, Ramchandani M, Landa D, Born T, Kaur P. Developing the totality of evidence for biosimilars: regulatory considerations and building confidence for the healthcare community. BioDrugs. 2017;31(3):175-87.

9. Hutterer KM, Polozova A, Kuhns S, McBride HJ, Cao $\mathrm{X}$, Liu J. Assessing analytical and functional similarity of proposed Amgen biosimilar ABP 980 to trastuzumab. BioDrugs. 2019;33(3):321-33.

10. European Medicines Agency. Committee for Medicinal Products and Human Use. Assessment Report. KANJINTI. 22 March 2018. https://www. ema.europa.eu/en/documents/assessment-report/ kanjinti-epar-public-assessment-report_en.pdf. Accessed 4 Nov 2019.

11. Jassem S, Wang W, Sweet $\mathrm{H}$, et al. Functional and nonclinical similarity of ABP 980, a biosimilar of trastuzumab. Pharm Res. 2019;36(12):177.

12. Hanes V, Born T, Chow V, et al. Comparative similarity of ABP 980 and trastuzumab: results of functional similarity and human pharmacokinetic assessment. In: Abstract 3147. Presented at San Antonio Breast Cancer Conference. 8-12 December 2015.

13. Kolberg HC, Colleoni M, Santi P, et al. Totality of scientific evidence in the development of ABP 980, a biosimilar to trastuzumab. Target Oncol. 2019;14(6):647-56.

14. Hanes V, Chow V, Zhang N, Markus R. A randomized, single-blind, single-dose study evaluating the pharmacokinetic equivalence of proposed biosimilar ABP 980 and trastuzumab in healthy male subjects. Cancer Chemother Pharmacol. 2017;79(5): 881-8.

15. von Minckwitz G, Colleoni M, Kolberg HC, et al. Efficacy and safety of ABP 980 compared with reference trastuzumab in women with HER2-positive early breast cancer (LILAC study): a randomised, double-blind, phase 3 trial. Lancet Oncol. 2018;19(7):987-98.

16. Kolberg HC, von Minckwitz G, Hanes V. Phase 3 LILAC study sets standard for clinical evaluation of oncology biosimilars. Oncotarget. 2019;10(1):8-9.

17. Cortes J, Curigliano G, Dieras V. Expert perspectives on biosimilar monoclonal antibodies in breast cancer. Breast Cancer Res Treat. 2014;144(2):233-9. 
18. Jackisch C, Scappaticci FA, Heinzmann D, et al. Neoadjuvant breast cancer treatment as a sensitive setting for trastuzumab biosimilar development and extrapolation. Future Oncol. 2015;11(1):61-71.

19. US Food and Drug Administration. Guidance for industry. Pathologic complete response in neoadjuvant treatment of high-risk early-stage breast cancer: use as an endpoint to support accelerated approval. July 2020. https://www.fda.gov/regu latory-information/search-fda-guidance-documents/ pathologic-complete-response-neoadjuvant-treatme nt-high-risk-early-stage-breast-cancer-use-endpoint. Accessed July 2020.

20. Kolberg HC, Colleoni M, Demetriou GS, et al. Cardiac safety of the trastuzumab biosimilar ABP 980 in women with HER2-positive early breast cancer in the randomized, double-blind, active-controlled LILAC study. Drug Saf. 2020;43(3):233-42.
21. Crampton S, Polozovab A, Asbury D. Stability of ABP 980, a trastuzumab biosimilar, after reconstitution of lyophilized drug product. Abstract 3147. In: Presented at 21st British Oncology Pharmacy Association (BOPA) Annual Symposium. 12-14 October 2018.

22. Wang W, Singh S, Zeng DL, King K, Nema S. Antibody structure, instability, and formulation. J Pharm Sci. 2007;96(1):1-26.

23. Crampton S, Polozova A, Asbury D, Lueras A, Breslin P, Hippenmeyer J, Litowski J, Goss M. Stability of the trastuzumab biosimilar ABP 980 compared to reference product after intravenous bag preparation, transport and storage at various temperatures, concentrations and stress conditions. Generics Biosimilars Initiative J (GaBI Journal). 2020;9(1): 5-13. https://doi.org/10.5639/gabij.2020.0901.002. 